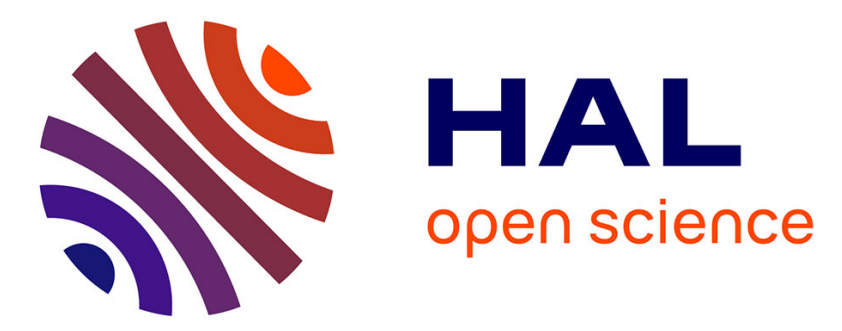

\title{
Yaglom-like equation in axisymmetric anisotropic turbulence
}

L. Danaila, J. F. Krawczynski, F. Thiesset, B F Renou

\section{To cite this version:}

L. Danaila, J. F. Krawczynski, F. Thiesset, B F Renou. Yaglom-like equation in axisymmetric anisotropic turbulence. Physica D: Nonlinear Phenomena, 2012, Small Scale Turbulence, 241 (3), pp.216-223. 10.1016/j.physd.2011.08.011 . hal-01429828

\section{HAL Id: hal-01429828 \\ https://hal.sorbonne-universite.fr/hal-01429828}

Submitted on 9 Jan 2017

HAL is a multi-disciplinary open access archive for the deposit and dissemination of scientific research documents, whether they are published or not. The documents may come from teaching and research institutions in France or abroad, or from public or private research centers.
L'archive ouverte pluridisciplinaire HAL, est destinée au dépôt et à la diffusion de documents scientifiques de niveau recherche, publiés ou non, émanant des établissements d'enseignement et de recherche français ou étrangers, des laboratoires publics ou privés. 


\title{
Yaglom-like equation in axisymmetric anisotropic turbulence
}

\author{
L. Danaila, J.F. Krawczynski ${ }^{1}$, F. Thiesset and B. Renou \\ CORIA UMR 6614, Université de Rouen, BP 1276801 Saint Etienne du Rouvray, \\ FRANCE
}

\begin{abstract}
A scale-by-scale kinetic energy budget equation is developed for inhomogeneous and anisotropic turbulence. This equation reduces to Yaglom's 4/3 law, under more strict assumptions. Experimental data obtained in the impact region of two opposed jets, in a multiple-opposed-jets flow, are used to partially validate the analytical development and to better characterize this complex flow. It is shown that the energy transfer is mainly performed in planes perpendicular to the axisymmetry axis, whereas it is strongly inhibited along the axisymmetry direction.
\end{abstract}

Keywords: scale-by-scale energy budgets, axisymmetric turbulence, coherent motion, opposed jets, PIV measurements, energy transfer

\section{General picture and isotropic context}

A lot of effort is being devoted to understanding and modelling real flows, whereas most of them are anisotropic and/or populated by coherent structures. The latter are often the result of initial or boundary conditions. Therefore, either the near field of decaying flows, or forced turbulence in small volumes, strong anisotropy is usually present. The issue addressed here is how to characterize these flows from an analytical viewpoint which concerns kinetic energy at a given scale.

Let us first briefly remind the existing analytical tools, developed in the context of isotropic and locally isotropic turbulence.

\footnotetext{
${ }^{1}$ Present address: UPMC Univ Paris 06, UMR 7190, Institut Jean Le Rond d'Alembert, F-75005 Paris, France.
} 
1 Strictly isotropic context

Kolmogorov's relationship [1], [2] between the second-order moment, $\left\langle(\delta u)^{2}\right\rangle$, of the velocity increment along the flow direction, $\delta u(r)=$ $u(x+r)-u(x)$, over an interval $r$ in the longitudinal $(x)$ direction and the third-order moment, $\left\langle(\delta u)^{3}\right\rangle$, is given by

$$
-\left\langle(\delta u)^{3}\right\rangle+6 \nu \frac{d}{d r}\left\langle(\delta u)^{2}\right\rangle=\frac{4}{5}\langle\epsilon\rangle r
$$

where $\langle\epsilon\rangle$ is the mean energy dissipation rate defined as

$$
\langle\epsilon\rangle=\frac{1}{2} \nu\left\langle\left(\frac{\partial u_{i}}{\partial x_{j}}+\frac{\partial u_{j}}{\partial x_{i}}\right)^{2}\right\rangle .
$$

Here, and throughout the paper, repeated indices indicate summation, $\nu$ is the kinematic viscosity of the fluid, $u_{i}$ is the fluctuating velocity component in the $i-t h$ direction, and angular brackets denote time averaging. Equation (1) is derived within a framework [1] which assumes a cascade that is universal and locally isotropic for small enough scales and large enough Reynolds numbers. When the effect of viscosity is negligible, Eq. (1) reduces to the so-called 'four-fifths law',

$$
-\left\langle(\delta u)^{3}\right\rangle=\frac{4}{5}\langle\epsilon\rangle r
$$

which has a cornerstone role in the study of turbulence, since it is the simplest result derived from relations expressing conservation of momentum, using homogeneity and local isotropy. Writing Eq. (1) symbolically as $A+B=C$, term $C$ - directly proportional to $\langle\epsilon\rangle$ - is associated with the transfer of energy at a scale $r$. Equation (1) indicates that, at each scale, energy is transferred by both turbulent advection (term $A$ ) and molecular diffusion (term $B$ ). Equations (1) and (3) have also been often used as alternate, experimental, means of determining $\langle\epsilon\rangle$ via measurements of $\left\langle(\delta u)^{2}\right\rangle$ and $\left\langle(\delta u)^{3}\right\rangle$.

At small Reynolds numbers, the sum $A+B$ cannot be expected to balance $C$, except at the smallest scales (e.g., for grid turbulence, this equality is satisfied only for $r / \eta \lesssim 5$ at $R_{\lambda}=66$ [3]). Here $R_{\lambda} \equiv u \lambda / \nu$, where $u$ is the root-mean-square of the longitudinal velocity fluctuation and $\lambda \equiv\left(\left\langle u^{2}\right\rangle /\left\langle(\partial u / \partial x)^{2}\right\rangle\right)^{\frac{1}{2}}$ is the longitudinal Taylor microscale. For 
intermediate Reynolds numbers $\left(100<R_{\lambda}<500\right)$, Eq. (1) is not satisfied for moderate to large scales, e.g. [4] [5]. This equation is only satisfied up to a separation which depends on the Reynolds number. Obviously, it cannot be verified at large $r$, when the contributions from the two terms on the left side of Eq. (1) become negligible.

In deriving Eq. (1) from the Kármán-Howarth equation [6], the nonstationarity term was ignored. Inclusion of this term would result in a transport equation for $\left\langle\left(\delta u^{2}\right)\right\rangle$ across all scales

$$
-\left\langle(\delta u)^{3}\right\rangle+6 \nu \frac{d}{d r}\left\langle(\delta u)^{2}\right\rangle-3 \frac{U}{r^{4}} \int_{0}^{r} s^{4} \frac{\partial}{\partial x}\left\langle(\delta u)^{2}\right\rangle d s=\frac{4}{5}\langle\epsilon\rangle r,
$$

where $U$ is the mean velocity and $s$ is a dummy variable. Equation (4) relates the second- to the third-order structure function of $u$ at all separations [7], [3, 8]. At sufficiently large $r$, relation (4) correctly represents the one-point energy budget for spatially decaying homogeneous isotropic turbulence, viz. $\langle\epsilon\rangle=-U \frac{3}{2} \frac{d}{d x}\left\langle u^{2}\right\rangle$. At large $r$, the extra term in Eq. (4), relative to Eq. (1), becomes the major contribution. This term arises because $\left\langle(\delta u)^{2}\right\rangle$ varies with $x$. This variation is due to the decaying large scales. In flows which are not homogeneous and isotropic, there may be other extra terms in Eq. (1), reflecting the large scale inhomogeneities, such as turbulent diffusion or production, and they may differ intrinsically between different flows. Indeed, the difference may be such that, for the same $R_{\lambda}$, the inhomogeneous contributions may vary from flow to flow or possibly different regions of the same flow.

At the smallest scales, Eq. (1) is consistent with the isotropic definition of $\langle\epsilon\rangle$, e.g.

$$
\langle\epsilon\rangle_{i s o}=15 \nu\left\langle\left(\frac{\partial u}{\partial x}\right)^{2}\right\rangle .
$$

This result is expected since Kolmogorov's equation is underpinned by the physical idea of a cascade of energy from large to small scales and an eventual decorrelation between the small (isotropic) scales and the large (anisotropic) scales. In the limit of $r \rightarrow 0$, Eq. (1) also leads (using a Taylor series development) to the transport equation of the enstrophy (or that for the mean energy dissipation rate $\langle\epsilon\rangle$ ) for isotropic turbulence [9]. 
2 Locally isotropic context

We mainly focus our attention on the following transport equation for $\left\langle\delta u_{i} \delta u_{i}\right\rangle \equiv\left\langle(\delta q)^{2}\right\rangle$, e.g. [10]

$$
-\left\langle\delta u(\delta q)^{2}\right\rangle+2 \nu \frac{d}{d r}\left\langle(\delta q)^{2}\right\rangle=\frac{4}{3}\langle\epsilon\rangle r,
$$

which is the analogous (in homogeneous isotropic turbulence) of the Yaglom equation [11] derived for temperature fluctuations. The termto-term analogy comes from the fact that both of them are transport equations of scalars (total kinetic energy and temperature variance at a given scale), whereas the coefficient in the right hand side (RHS) is 4/3. For very small scales, Eq. (6) complies with another form of $\langle\epsilon\rangle$, viz. $[12]$

$$
\langle\epsilon\rangle_{q}=3 \nu\left\langle\frac{\partial u_{i}}{\partial x} \frac{\partial u_{i}}{\partial x}\right\rangle .
$$

The non-negligible differences between the statistics of $u$ and the other two velocity components in most of the (anisotropic) flows justifies using $\delta u_{i}$ instead of $\delta u$. Following from Eq. (7), another definition of the Taylor microscale is $\lambda=\left(5 \nu\left\langle u_{i} u_{i}\right\rangle /\langle\epsilon\rangle_{q}\right)^{1 / 2}$, from which $R_{\lambda} \equiv$ $\left(\left\langle u_{i} u_{i}\right\rangle / 3\right)^{1 / 2} \lambda / \nu$. These forms will be used in the following.

Because most of the flows are anisotropic, at least over a range of scales, the far aim of this work is to understand, at a given scale, how energy is transferred, produced, diffused and dissipated, in anisotropic flows. bf First, we develop an analytical tool which can be used to characterize in real space, the turbulent cascade. This tool is specific to anisotropic axisymmetric flows. Second, this paper is aimed at quantifying anisotropic energy transfer along different spatial directions, in the impact region among two opposed jets with lateral fluid confinement.

Section 2 is devoted to the analytical development, in the general context 2.1 and in anisotropic context 2.2. Section 3 deals with a brief description of the experimental set-up and of the measurements. The mean flow is characterized in 3.2 and the fluctuating field in 3.3. Results are discussed in section 4 . 


\section{Analytical development}

\subsection{Scale-by-scale energy budget equation}

Using the same procedure as outlined in $[13,14,2]$, we write the incompressible Navier-Stokes equations at the two points $\vec{x}$ and $\vec{x}^{+}$, which are separated by the increment $\vec{r}=\vec{x}^{+}-\vec{x}$, viz.

$$
\begin{array}{r}
\partial_{t} u_{i}+U_{\alpha} \partial_{\alpha} u_{i}+u_{\alpha} \partial_{\alpha} U_{i}+U_{\alpha} \partial_{\alpha} U_{i}+u_{\alpha} \partial_{\alpha} u_{i}= \\
-\partial_{i}(p+P) / \rho+\nu \partial_{\alpha}^{2}\left(u_{i}+U_{i}\right) \\
\partial_{t} u_{i}^{+}+U_{\alpha}^{+} \partial_{\alpha} u_{i}^{+}+u_{\alpha}^{+} \partial_{\alpha} U_{i}^{+}+U_{\alpha}^{+} \partial_{\alpha} U_{i}^{+}+u_{\alpha}^{+} \partial_{\alpha}^{+} u_{i}^{+}= \\
-\partial_{i}^{+}\left(p^{+}+P^{+}\right) / \rho+\nu \partial_{\alpha}^{2+}\left(u_{i}^{+}+U_{i}^{+}\right),
\end{array}
$$

where $u_{i}$ and $U_{i}$ denote the fluctuating velocity, i.e. $\left\langle u_{i}\right\rangle=0$, and the mean velocity respectively. The mean velocity field is considered as being stationary. $p$ is the fluctuating pressure and $P$ is the mean pressure. The superscript + refers to $\vec{x}^{+}$and $\rho$ is the fluid density. In (8) and (9) $\partial_{t} \equiv \partial / \partial t, \partial_{\alpha} \equiv \partial / \partial x_{\alpha}$ and $\partial_{\alpha}^{2}$ is the Laplacian $\partial^{2} / \partial x_{\alpha}^{2}$ (hereafter, the notation $\partial_{\alpha}$ and $\partial_{\alpha}^{+}$will be used to denote derivatives with respect to $x_{\alpha}$ and $x_{\alpha}^{+}$; when other spatial variables are involved, the derivatives will be written explicitly, e.g., $\partial / \partial r_{\alpha}$ or $\left.\partial / \partial X_{\alpha}\right)$. We then consider that the two points $\vec{x}$ and $\vec{x}^{+}$are independent, i.e. $u_{i}$ depends only on $\vec{x}$ and $u_{i}^{+}$depends only on $\vec{x}^{+}$.

Under these conditions, subtraction of (8) from (9) yields an equation for the fluctuating velocity increment $\delta u_{i}=u_{i}^{+}-u_{i}$ (increments of any arbitrary function $\mathrm{f}$ are defined as $\left.\delta f \equiv f^{+}-f\right)$, viz.

$$
\begin{array}{r}
\partial_{t}\left(\delta u_{i}\right)+\delta\left(U_{\alpha} \partial_{\alpha} u_{i}\right)+\delta\left(u_{\alpha} \partial_{\alpha} U_{i}\right) \\
+\delta\left(U_{\alpha} \partial_{\alpha} U_{i}\right)+u_{\alpha}^{+} \partial_{\alpha}^{+}\left(\delta u_{i}\right)+u_{\alpha} \partial_{\alpha}\left(\delta u_{i}\right)= \\
-\left(\partial_{i}+\partial_{i}^{+}\right)(\delta(p+P)) / \rho+\nu\left(\partial_{\alpha}^{2}+\partial_{\alpha}^{2+}\right)\left(\delta\left(u_{i}+U_{i}\right) .\right.
\end{array}
$$

Following the approach suggested by [13], we also consider the gradient with respect to the midpoint, i.e.

$$
\vec{X}=\frac{1}{2}\left(\vec{x}+\vec{x}^{+}\right)
$$


Thus,

$$
\begin{gathered}
\partial_{\alpha}^{+} \equiv \frac{\partial}{\partial r_{\alpha}}+\frac{1}{2} \partial_{X_{\alpha}} \\
\partial_{\alpha} \equiv-\frac{\partial}{\partial r_{\alpha}}+\frac{1}{2} \partial_{X_{\alpha}}
\end{gathered}
$$

resulting in $\partial_{X_{\alpha}}=\partial_{\alpha}+\partial_{\alpha}^{+}$.

By taking into account (12), multiplying Eq. (10) by $2 \delta u_{i}$, averaging and some calculations, we finally obtain

$$
\begin{array}{r}
\partial_{t}\left\langle(\delta q)^{2}\right\rangle(\vec{r})+2\left\langle\delta\left(U_{\alpha} \partial_{\alpha} u_{i}\right) \cdot \delta u_{i}\right\rangle(\vec{r})+2\left\langle\delta\left(u_{\alpha} \partial_{\alpha} U_{i}\right) \cdot \delta u_{i}\right\rangle(\vec{r}) \\
+\frac{1}{2}\left\langle\left[\partial_{\alpha}+\partial_{\alpha}^{+}\right] \cdot\left[u_{\alpha}+u_{\alpha}^{+}\right](\delta q)^{2}\right\rangle \\
+2 / \rho\left(\partial_{i}+\partial_{i}^{+}\right)\left\langle\delta p \cdot \delta u_{i}\right\rangle(\vec{r})+\frac{\partial}{\partial r_{\alpha}}\left\langle\delta u_{\alpha}(\delta q)^{2}\right\rangle(\vec{r})= \\
+2 \nu \frac{\partial^{2}}{\partial r_{\alpha}{ }^{2}}\left\langle(\delta q)^{2}\right\rangle(\vec{r})-2\left(\langle\epsilon\rangle+\langle\epsilon\rangle^{+}\right) .
\end{array}
$$

Note that the first four terms correspond to large-scale effects. In (14), each term depends on the spatial vector $\vec{r}$. Therefore, Eq. (14) formally writes

$$
\begin{array}{r}
D(\vec{r})+T(\vec{r})+P(\vec{r})+T D(\vec{r})+P D(\vec{r}) \\
+\frac{\partial}{\partial r_{\alpha}}\left\langle\delta u_{\alpha}(\delta q)^{2}\right\rangle(\vec{r})=2 \nu \frac{\partial^{2}}{\partial r_{\alpha}{ }^{2}}\left\langle(\delta q)^{2}\right\rangle(\vec{r})-2\left(\langle\epsilon\rangle+\langle\epsilon\rangle^{+}\right),
\end{array}
$$

where terms $T, P, T D$ and $P D$ are the transport, production, turbulence diffusion and pressure diffusion terms, respectively.

\subsection{Axisymmetric context}

An anisotropic (axisymmetric) treatment is to be further applied to equation (15), in which each term depends on the spatial vector $\vec{r}$. Note also that this is a scalar equation, representing the scale-by-scale kinetic energy. Let us assume that the flow is axisymmetric with respect to a direction specified by $\vec{n}$. Flow statistics are then invariant to rotations in planes normal to $\vec{n}$ and symmetric with respect to $\vec{n}$. We note

$$
r^{2}=\vec{r} \cdot \vec{r} \quad \text { and } \quad r \mu \equiv \vec{r} \cdot \vec{n} .
$$


In this context, each term of the equations depends on two variables, $r$ and $\mu$. Large-scale inhomogeneous variations might be observed along the axisymmetry direction $\vec{n}$. We consider that statistics are homogeneous in planes perpendicular to $\vec{n}$.

The advection term $\frac{\partial}{\partial r_{\alpha}}\left\langle\delta u_{\alpha}(\delta q)^{2}\right\rangle(\vec{r})$ is written in a manner similar to [15] and [16]:

$$
\left\langle\delta u_{\alpha}(\delta q)^{2}\right\rangle(\vec{r})=M_{a}(r, \mu) r_{\alpha}+N_{a}(r, \mu) n_{\alpha},
$$

where only two scalars $M_{a}$ and $N_{a}$ appear (the subscript $a$ stands for the 'advection' term in the kinetic energy budget equation). A very important remark is that the scalars $M_{a}(r, \mu)$ and $N_{a}(r, \mu)$ can be determined from planar experiments which include the axisymmetry vector $\vec{n}$. For instance, PIV (Particle Image Velocimetry) measurements in planes including $\vec{n}$, provide velocity fluctuations $u_{\|}$and $u_{\perp}$, parallel and perpendicular to $\vec{n}$. Therefore, by supposing axisymmetry for the third (unmeasured) velocity component, quantities such as $\left\langle\delta u_{\|}(\delta q)^{2}\right\rangle$ and $\left\langle\delta u_{\perp}(\delta q)^{2}\right\rangle$ are measurable. This leads to the experimentally determinable scalars:

$$
\begin{array}{r}
M_{a}(r, \mu)=\frac{\left\langle\delta u_{\perp}(\delta q)^{2}\right\rangle(r, \mu)}{r \sqrt{\left(1-\mu^{2}\right)}} \\
N_{a}(r, \mu)=\left\langle\delta u_{\|}(\delta q)^{2}\right\rangle(r, \mu)-\left\langle\delta u_{\perp}(\delta q)^{2}\right\rangle(r, \mu) \frac{\mu}{\sqrt{1-\mu^{2}}}
\end{array}
$$

Therefore, for the axisymmetric case, $\frac{\partial}{\partial r_{\alpha}}\left\langle\delta u_{\alpha}(\delta q)^{2}\right\rangle(\vec{r})$ becomes

$$
\frac{\partial}{\partial r_{\alpha}}\left\langle\delta u_{\alpha}(\delta q)^{2}\right\rangle(\vec{r}) \equiv\left(r \frac{\partial}{\partial r}+3\right) M_{a}(r, \mu)+\left(\mu \frac{\partial}{\partial r}+\frac{1-\mu^{2}}{r} \frac{\partial}{\partial \mu}\right) N_{a}(r, \mu) .(19
$$

The dissipative term $\operatorname{Diss}(r, \mu)$ is to be further explicited by using the axisymmetric form of the Laplacian $\Delta(r, \mu)$ [17], [18].

Equation (15) can therefore be written formally as

$$
\begin{array}{r}
D(r, \mu)+T(r, \mu)+P(r, \mu)+T D(r, \mu)+P D(r, \mu)+ \\
\left(r \frac{\partial}{\partial r}+3\right) M_{a}(r, \mu)+\left(\mu \frac{\partial}{\partial r}+\frac{1-\mu^{2}}{r} \frac{\partial}{\partial \mu}\right) N_{a}(r, \mu)= \\
2 \nu \Delta(r, \mu)\left\langle\delta q^{2}\right\rangle-2\left[\langle\epsilon\rangle+\langle\epsilon\rangle^{+}\right] .
\end{array}
$$

Finally, in order to simplify the mathematical form of this equation, we introduce $\mathcal{V}$ such that $N_{a}(r, \mu)=\left[2+r \frac{\partial}{\partial r}\right] \mathcal{V}=\frac{1}{r} \frac{\partial}{\partial r}\left[r^{2} \mathcal{V}\right]$, with $\mathcal{V}=\frac{1}{r^{2}} \int_{0}^{r} s N_{a}(s, \mu) \mathrm{d} s$ 
where $s$ is a dummy variable. We further define

$$
N_{a}^{*}=\left(\mu \frac{\partial}{\partial r}+\frac{1-\mu^{2}}{r} \frac{\partial}{\partial \mu}\right) \mathcal{V}
$$

which is obviously determinable from experiments, so that Eq. (20) reduces to

$$
\begin{array}{r}
D(r, \mu)+T(r, \mu)+P(r, \mu)+T D(r, \mu)+P D(r, \mu)+ \\
\left(r \frac{\partial}{\partial r}+3\right)\left(M_{a}(r, \mu)+N_{a}^{*}(r, \mu)\right)= \\
2 \nu \Delta(r, \mu)\left\langle(\delta q)^{2}\right\rangle(r, \mu)-2\left[\langle\epsilon\rangle+\langle\epsilon\rangle^{+}\right] .
\end{array}
$$

By noting $G=M_{a}(r, \mu)+N_{a}^{*}(r, \mu)$ and $\left(r \frac{\partial}{\partial r}+3\right)=\frac{1}{r^{2}} \frac{\partial}{\partial r}\left(r^{3}\right)$, and after integration with respect to $r$, the final axisymmetric form of scale-by-scale energy budget equation is (also discussed in the context of a parallel with the spectral space developments in [18])

$$
\begin{array}{r}
G(r, \mu)=-\frac{2}{3}\left[\langle\epsilon\rangle+\langle\epsilon\rangle^{+}\right]+ \\
2 \nu \frac{1}{r^{3}} \int_{0}^{r} s^{2} \Delta(r, \mu)\left\langle(\delta q)^{2}\right\rangle \mathrm{d} s \\
-\frac{1}{r^{3}} \int_{0}^{r} s^{2}(D(r, \mu)+T(r, \mu)+P(r, \mu)+T D(r, \mu)+P D(r, \mu)) \mathrm{d} s
\end{array}
$$

Note the importance of (23) with respect to the classical known equations:

- it obviously reduces to the isotropic ' $4 / 3$ ' law ;

- it contains large-scale terms (decay, transport, production etc.) which are very important in the context of the validation against experimental data;

- it contains an explicit spatial variation of the mean kinetic energy dissipation rate.

- it takes into account both inhomogeneity and anisotropy (though, under the assumption of axisymmetry). 
In the following, we investigate (experimentally) the reduced form of this equation:

$$
G(r, \mu)=-\frac{2}{3}\left[\langle\epsilon\rangle+\langle\epsilon\rangle^{+}\right]
$$

which comes to study the (total) kinetic energy transferred at each scale, by velocity fluctuations. Equation (24) is the analogous of Yaglom's equation in anisotropic, axisymmetric turbulence.

\section{Brief description of the flow generated by multiple opposed jets}

The experiments were conducted in the confined-opposed-jets chamber, Fig. 1, in which the work of [19] was previously carried out. Particular to this chamber is that 16 pairs of opposed jets coexist and interact with their characteristic instabilities, thus leading to their local confinement. Therefore, the basic flow pattern is a pair of opposed, confined, jets.

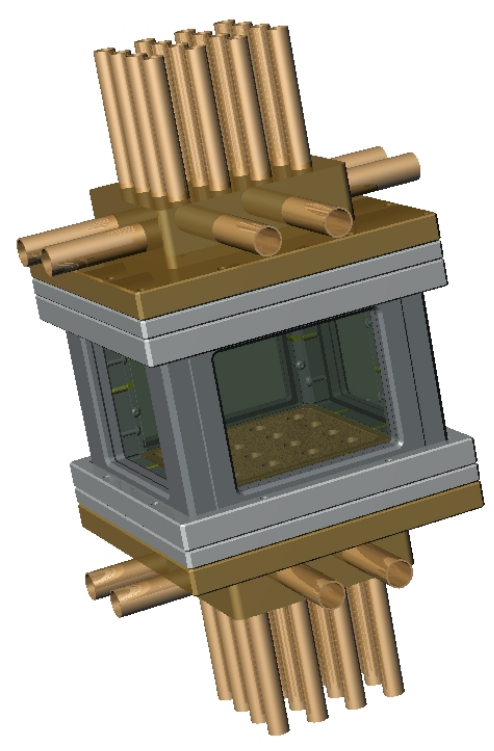

Figure 1: Experimental set-up.

Turbulence generation, the interaction with large-scale component of the flow, and the local confinement induce inherent anisotropy. Although the particular geometrical features of this chamber generate successive anisotropic 
energy injection, it was shown that, towards the exit from the reactor, the velocity fluctuations become nearly homogeneous and isotropic [19].

At present, we turn our attention to the region responsible for the velocity fluctuations generation, i.e. the impact region among each two opposed jets. As a result of collisions between confined opposed streams and their subsequent instabilities, a relatively narrow zone of intense velocity fluctuations is generated, which offers a priori excellent conditions for intensifying mixing rate.

\subsection{Apparatus and measurements}

The test section consisted of a rectangular parallelepiped $(110 \times 110 \times$ $\left.60 \mathrm{~mm}^{3}\right)$ equipped with quartz Suprasil windows $\left(100 \times 80 \mathrm{~mm}^{2}\right)$ on each side. Both the inlet and the outlet of the fluid were located on the top and bottom boundary plates made of a $24 \mathrm{~mm}$-spacing square-matrix arrangement of jets and of a porous, homogeneous, media in between them. The porous boundary plates were backed by plena connected to the exhaust piping network. $200 \mathrm{~mm}$-long tubes were supplying the 16 pairs of jets issuing from the top and bottom boundary plates. Air mass flow-rates were controlled by two Bronkhorst (EL-FLOW) mass flow-meters whose accuracy was estimated at $\pm 0.5 \%$ of the full scale (100 NCMH, normal cube meters per hour). Micrometric valves were used to match the flow rate issuing from every individual jet. The inner-chamber static pressure as well as the pressure drop of each exhaust plena could be adjusted with two regulating valves connected to the exhaust piping network. One wall was equipped with a $1 \mathrm{~mm}$-diameter pressure tap, connected to a digital manometer. A static pressure transducer (Keller) was used to measure the mean flow pressure with a resolution of 1 mbar and an accuracy of 0.03 bars. The geometry of the facility is characterized by the following outer scales: the horizontal mid-distance between two consecutive jet-axes, $L=12 \mathrm{~mm}$ kept fixed, the vertical mid-distance between two opposed jets, $h=30 \mathrm{~mm}$ and the inner jet diameter, $d_{j}=10$ $\mathrm{mm}$ or $d_{j}=6 \mathrm{~mm}$.

The instantaneous, two-dimensional velocity measurements relied on Particle Image Velocimetry (PIV) with DEHS (Di-Ethyl-Hexyl-Sebacat, $\mathrm{C}_{26} \mathrm{H}_{50} \mathrm{O}_{4}$ ) particles $\left(\rho_{p}=918 \mathrm{~kg} / \mathrm{m}^{3}\right)$. The light source was a Nd-Yag laser (Big Sky laser, $120 \mathrm{~mJ} /$ pulse) with a second-harmonic-generating crystal producing a Q-switched laser output in the green $(532 \mathrm{~nm})$. Light scattered from the particles was collected on a CCD camera (FlowMaster La Vision, 12 bits, $1280 \times 1024$ pix $^{2}$ ) with a $50 \mathrm{~mm} \mathrm{f} / 1.2$ Nikkor lens. The optical arrangement 
yielded a magnification of $20.5 \mathrm{pix} / \mathrm{mm}$, calibrated using images of a precision reference grid. PIV processing was performed with a cross-correlation technique between pairs of successive images. The initial size of the PIV interrogation window was $64 \mathrm{pix}^{2}$. Six iterations were used to obtain a final interrogation window size of $16 \mathrm{pix}^{2}$, with a $50 \%$ overlap. The time interval between two consecutive images was in the range $3-8 \mu \mathrm{s}$, scaled approximately inversely with the injection velocity, $V_{i n j}$. The determination of the minimum fully-resolved length-scale was discussed in details in [19]. An analysis based on the estimation of the PIV spectral transfer-function [20] yielded to a cut-off length scale due to the low-pass filtering effect of our PIV system of $\lambda_{c}=1 / k_{c} \simeq 1.7 \mathrm{~mm}$. The spatial resolution, as well as the largest measurable scales, are fixed for all the experiments by the optics, the CCD focal-plane array resolution, and the PIV processing, and are independent of the flow.

\subsection{Flow overview}

The flow description is based on planar cuts in the jet-axis plane (parallel to the $O X Y$-plane), see Fig. 2a that illustrates the flow resulting from four pairs of opposed jets. Here, $u$ is the velocity along the horizontal $x$ direction, and $v$ along the vertical $y$ direction, i.e. parallel to the jets axes. Arrows in Fig. 2a represent schematic trajectories of particles transported by the mean velocity field only, see Fig. 2b. Statistics discussed below are based on time-averaging performed in each image pixel over a set of 1500 images.

Figure $2 \mathrm{~b}$ represents the mean velocity field, $V_{\text {total }}=\left(U^{2}+V^{2}\right)^{0.5}$, normalized by the injection velocity $V_{i n j}$, for two pairs of opposed jets. We choose to further investigate this flow pattern, by keeping in mind the periodicity and symmetry of this flow that are well-captured by the experimental implementation. The impingement of symmetric opposed jets generates stagnation points, the first one (noted as [1] on Fig. 2 b) is present between two opposed jets. Particular to this flow is the local confinement of every pair of opposed jets that constraints the radial flow issuing from their collision, thus creating a secondary statistically stagnation point at mid-horizontal-distance between two pairs of opposed jets (point [2] on Fig. 2 b). The third point noted on the same figure is [1'], placed between the two pairs of opposed jets.

The stagnation region between two opposed-axial-jets is characterized by strong variations of the velocity field in the $y$ direction and by nearly axisymmetrical statistical properties [19]. The strong variations over very 


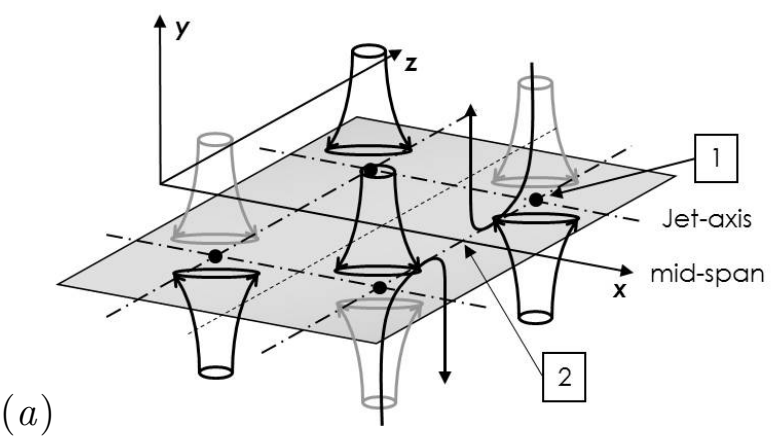

(b)



Figure 2: (a): Schematic of the flow. (b): Normalized mean velocity field, $V_{t o t a l} / V_{i n j}$, in the jet-axis plane, as function of $x / d_{j}$ and $y / d_{j}$.

short distances leads to energy injection over a direction perpendicular to the instantaneous stagnation plane, thus not necessarily parallel to $y$. Fluid laterally escapes in the local, instantaneous, stagnation plane and returns towards exits. The interaction between fluid injected and the return flow, along with the local-confinement effects, produce large scale annular shearlayers, see Fig. 5 in

\subsection{Fluctuating flow and mean energy dissipation}

Noteworthy is the unstable aspect of the flow which significantly contrasts with the mean flow aspect and its remarkable symmetries. Instantaneous images as depicted in Fig. 3 suggest up-and-down and left-right movements of the very thin fluid interface among each two opposed jets, which is generally not parallel to the mean stagnation plane. [19]. Instabilities 
$(a)$



(b)

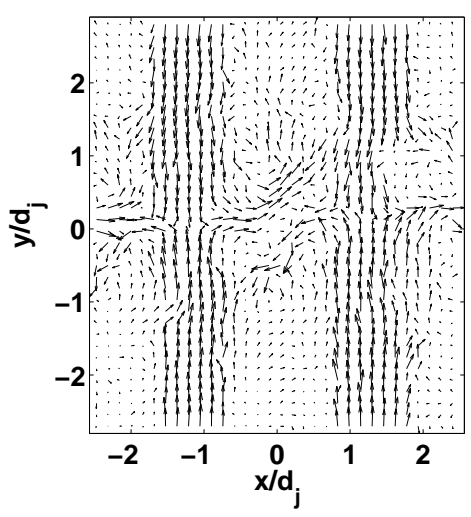

Figure 3: Instantaneous velocity vectors field, in the jet-axis plane, as function of $x / d_{j}$ and $y / d_{j}$.

which are present here are a subtle combination of opposed jets instabilities $[21,22,23,24]$, twin-jets instabilities [25, 26] and one confined-jet with backflow [27]. Therefore, obtaining a mean stagnation plane region, i.e. all the stagnation points being aligned on the same horizontal plane, represents a challenge in the present experiment and requires a detailed balance for the mass flow issuing through each of the 32 injecting jets.

Velocity fluctuations are mainly produced during strong variations of the mean velocity in the stagnation region. In between two axially opposed-jets, the most intense strain is along $y$. This results in a significant anisotropy level in this region, as illustrated for instance by the ratio $\left\langle u^{2}\right\rangle^{1 / 2} /\left\langle v^{2}\right\rangle^{1 / 2}$, represented on Fig. 4, which is much smaller than the isotropic value of 1. In the stagnation point [2], fluctuations along the horizontal direction are mainly created, and therefore the above-mentioned ratio becomes larger than 1.

Therefore, most of the total kinetic energy, calculated using axisymmetry hypothesis, i.e. $\left\langle q^{2}\right\rangle=2 \times\left\langle u^{2}\right\rangle+\left\langle v^{2}\right\rangle$, is concentrated in the impinging region of the confined chamber, see Fig. 5. This is a particularity of the stagnation flow among two fluids, for which the stagnation point is both associated to a stagnation of the mean velocity and a maximum of velocity fluctuations.

Our aim is to assess Eq. (24) in the region around the stagnation point [1] among two opposed jets, where a lot of kinetic energy is present. It is therefore necessary to determine properly the mean energy dissipation 




Figure 4: Ratio $\left\langle u^{2}\right\rangle^{1 / 2} /\left\langle v^{2}\right\rangle^{1 / 2}$ in the jet-axis plane.

rate in that region. This is not an easy task in this flow, and with PIV measurements. The values of the mean energy dissipation rate $\langle\epsilon\rangle$ which were determined with the methods widely described and discussed in [19]. The distribution is shown on Fig. 6. Important values of $\langle\epsilon\rangle$ are present in stagnation points [1] and [1'], but the most important values are noted in the central, stagnation point [2], where turbulence has had time to develop, and where turbulent/small scale kinetic energy (not shown here) is the most important.

In the following, with this spatial distribution of $\langle\epsilon\rangle$, we proceed to testing Eq. (24).

\section{Results. Anisotropic energy transfer in the impact region of two opposed jets with counterflow}

This section is devoted to the experimental investigation of Eq. (24).

It is of interest to first present the distribution of the second-order structure functions $\left\langle(\delta q)^{2}\right\rangle$ as function of the Cartesian coordinates $r_{x}, r_{y}$ and afterwards of the cylindrical coordinates $r, \mu$, where $r_{x}$ and $r_{y}$ are the separations in the $x$ and $y$ direction. Therefore $r=\sqrt{r_{x}^{2}+r_{y}^{2}}$ and $\mu=\cos \left(\operatorname{Arctan}\left(\frac{r_{y}}{r_{x}}\right)\right)$. These structure functions are calculated by fixing the origin of the Cartesian coordinate system $\left(r_{x}, r_{y}\right)$ at the intersection of the axisymmetry axis of two opposed jets (and we fix here the left side pair of opposed jets) with the plane where the mean velocity is zero $V=U=0$ (point [1] on Fig. 2 (b)). Then, 


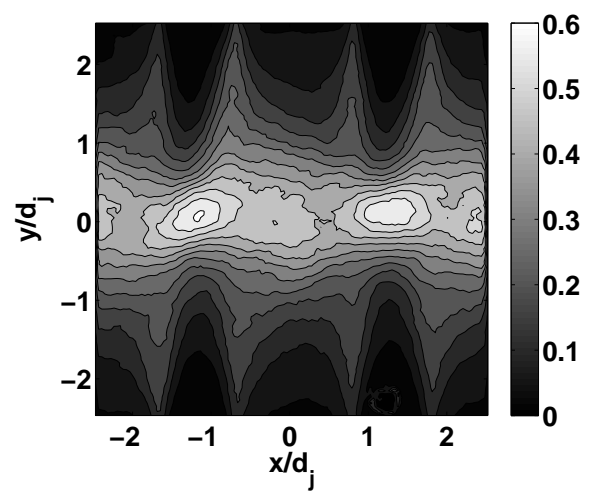

Figure 5: Distribution of the normalized total kinetic energy $\left\langle u_{i} u_{i}\right\rangle / V_{i n j}^{2}$. Filled contour plot in increments of 0.1 .

increments $\delta u_{i}$ are calculated

$$
\delta u_{i} \equiv u_{i}\left(r_{x}, r_{y}\right)-u_{i}\left(r_{x}=0, r_{y}=0\right) .
$$

Second-order structure functions, defined as $\left\langle\delta q^{2}\right\rangle \equiv\left\langle\left(\delta u_{i}\right)^{2}\right\rangle\left(r_{x}, r_{y}\right)$, are represented on Fig. 7 (left). It is obvious that in point $r_{x}=0, r_{y}=0$, the values of $\left\langle\delta q^{2}\right\rangle$ are zero. This function is different from zero at different positions in jets-axis plane. For instance, over the radial direction $r_{x}$, energy increases for increasing separations, exhibits a maximum in the stagnation point [2] created by the two pairs of jets, it further decreases in a region with less fluctuations and increases again in the stagnation point corresponding to the right-side opposed jets, [1']. Over the vertical direction $r_{y}$, energy increases but much less than over the radial/horizontal direction. Variations of energy over Fig. 7 (left) are to be correlated with the distribution of the total kinetic energy, as represented on Fig. 5.

Figure 7 (b) represents the same quantity $\left\langle\delta q^{2}\right\rangle$ but in $(r, \mu)$ coordinates. The axis $\mu=0$, for any value of $r$, physically corresponds to the horizontal axis $r_{x}$. Along this axis, the same behaviour of $\left\langle\delta q^{2}\right\rangle$ as previously discussed is to be emphasized: energy maxima are present for the two stagnation points of the flow: point [2], and the stagnation point among the two pairs of opposed jets. For $\mu= \pm 1$, corresponding to the vertical axis $r_{y}$, energy increase is to be noted, but in a much less quantity than over the radial direction (which is 'populated' with stagnation points). 


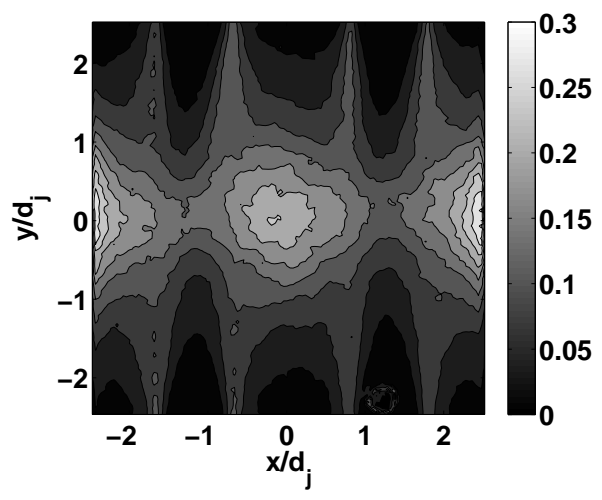

Figure 6: Distribution of the mean energy dissipation rate (normalized by $V_{i n j}^{3} / d_{j}$ ).

Figure 8 represents the distribution of $\langle\epsilon\rangle$ as a function of $(r, \mu)$. The same procedure as developed for $\left\langle(\delta q)^{2}\right\rangle$ was used. For $\mu=0$, corresponding to $\theta=90^{\circ}$ and therefore to the horizontal axis $r_{x}$, the dissipation increases in the stagnation point [2], then decreases, and further increases again (but to a less important value) towards the stagnation point [1'].

Finally, we represent term $G$ as a function of $(r, \mu)$, on Fig. 9. Term $G$ has the physical signification of the energy transferred by fluctuations at a given scale $r$, and a spatial position $r, \mu$. To our knowledge, this is for the first time when such anisotropic energy transfer, equivalent to $-\left\langle(\delta u)^{3}\right\rangle$ or to $-\left\langle\delta u(\delta q)^{2}\right\rangle$ in strictly isotropic turbulence, is obtained from experimental data. For $\mu=0$, term $G$ is first negative for scales smaller than the integral scale, corresponding to a classical direct cascade. It becomes positive for scales larger than the integral scale (beyond the stagnation point [2]), where the flow becomes strongly inhomogeneous. This change in sign does not signify an inverse cascade, but it is rather the signature of large-scale inhomogeneity. A similar behaviour is to be noted for values of $\mu$ nearly close to 0, i.e. around the horizontal direction $r_{x}$. This similarity is associated to the fact that in the central stagnation region, the mean velocity components are nearly equal to zero, and statistics closely satisfy local isotropy (over a limited range of scales). For values of $\mu= \pm 1$, corresponding to the vertical axisymmetry axis, term $G$ is much less important, over the whole range of scales, thus signifying that the energy transfer through total velocity fluctuations is strongly inhibited. This is associated to the fact that over this 


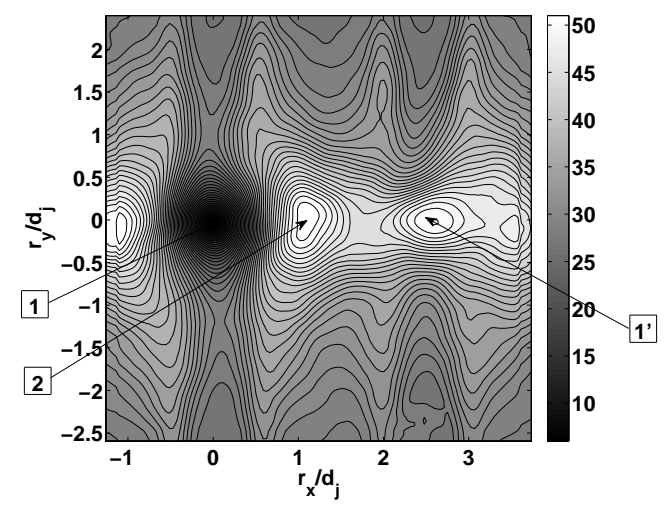

(b)

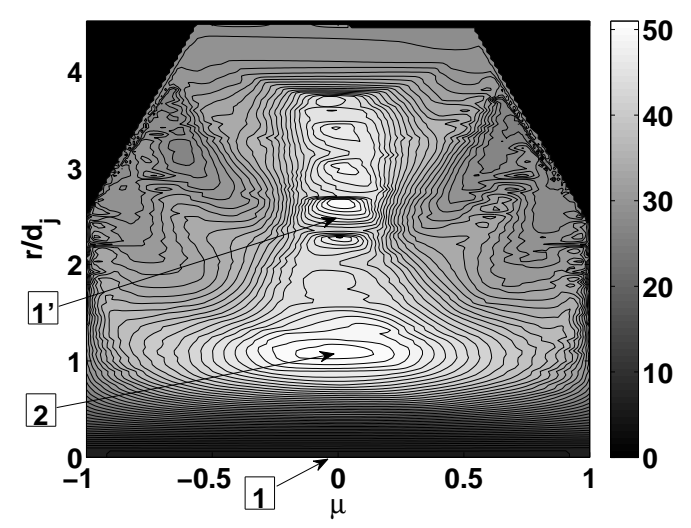

Figure 7: Distribution of the total kinetic energy at each scale $\left\langle\delta q^{2}\right\rangle$, when the first point is (1). (left) $\left\langle\delta q^{2}\right\rangle$ as function of $r_{x}, r_{y}$, (right) $\left\langle\delta q^{2}\right\rangle$ as function of $r, \mu$.

direction, kinetic energy is much less present (see Fig. 5).

The final budget, as given by Eq. (24), for this Reynolds number based on the Taylor microscale equal to 250, is represented on Fig. 9, right. Along the vertical direction $(\mu=1$, solid line and $\square$ ), the budget is not at all equilibrated, and the energy transfer term $G$ is much less important than the total energy transferred.

For an intermediate direction $(\mu=0.5$, dashed line and $\circ)$, the energy transfer is intensified, but it is still much smaller than the total energy transferred, as given by the RHS of Eq. (24).

Along the horizontal direction $(\mu=0$, dashed dotted lines and +$)$, and for small scales, the budget is best balanced, but not equilibrated mainly be- 


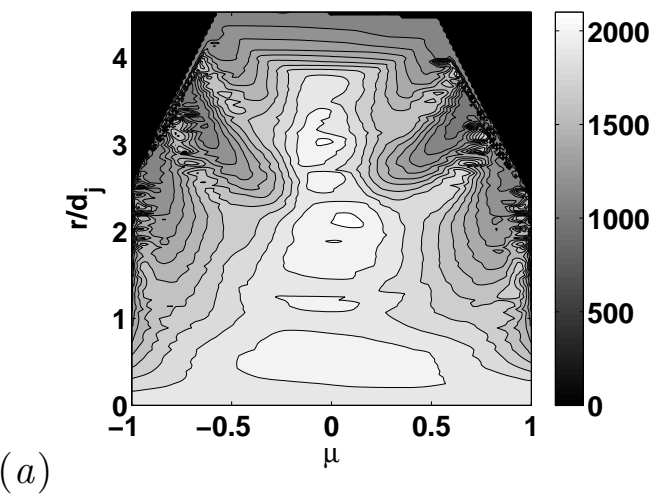

Figure 8: Distribution of term $\langle\epsilon\rangle+\langle\epsilon\rangle^{+}$in $r, \mu$ coordinates.

cause the molecular term is absent (this term is not represented here because PIV is not at all an adequate tool to calculate derivatives, and even less laplacians). For intermediate scales, the budget is better equilibrated, signifying that energy transferred by fluctuations equilibrates by itself the total energy transferred, and that other effects (shear, pressure-diffusion, decay etc.) are not important over this range of scales.

\section{Conclusions and perspectives}

We have presented the salient steps in obtaining an anisotropic, axisymmetric, scale-by-scale energy budget equation. The equation is developed for the kinetic energy, which is a scalar, therefore the calculations are much facilitated than those for the velocity field itself [28], [29], [30], [31]. The analytical development is similar to that of [16], but we take care of large-scale inhomogeneous terms, and we also consider spatial variations of the mean kinetic energy dissipation rate, without which the equation would have been mathematically inconsistent (left-hand-side would depend on $(r, \mu)$, but not the right-hand-side).

The reduced form of this equation has been used in order to have a deeper insight in the physics associated to two opposed jets, present in a multiple-opposed-jets reactor. We have proved that energy transfer along the horizontal direction is much more important than that along the axisymmetry direction. This behavior is associated to the quantity of kinetic energy 

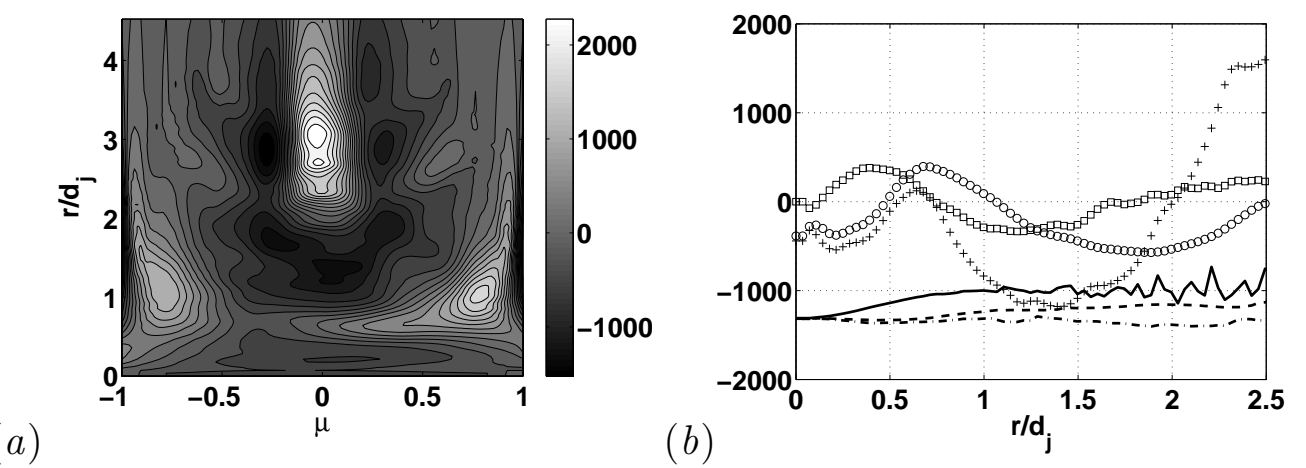

Figure 9: (a): Distribution of term $G$; (b): Eq. (24) for different values of $\mu=1$ (LHS: $\square$, RHS: solid line), $\mu=1 / 2$ (LHS: o , RHS: dashed line) and $\mu=0$ (LHS: + , RHS: dash-dotted line).

present along each direction, much more important along the horizontal one than along the vertical one.

Equation (24) can to be used as a tool to investigate the degree of anisotropy of different flows, and the manner in which different phenomena may affect the energy cascade. Open perspectives concern a similar development for flows in which other effects are present, such as rotation or stratification [32]. Moreover, the theory is developed for incompressible turbulence in homogeneous fluids (i.e. with uniform physical properties: constant density, viscosity etc.). A similar approach can obviously be developed for variable-properties turbulence, for which all the involved terms exhibit specific analytical forms (see for instance the book by Sagaut and Cambon [33], for expressions of the dissipation in compressible turbulence). The complexity of these terms lead to the necessity to use rather numerical simulations to perform this investigation, instead of under-resolved (in space/time) PIV. This issue is left for future.

Financial support from the French National Research Agency, under the projects 'Micromélange' and 'ANISO', is gratefully acknowledged. Professor P.E. Dimotakis is thanked for useful discussions. 


\section{References}

[1] A. Kolmogorov, Dokl. Akad. Nauk. SSSR 32 (1941).

[2] A. S. Monin, A. M. Yaglom, Statistical fluid mechanics, volume 2, MIT Press (ed. J. Lumley), Cambridge, Mass., 1975.

[3] L. Danaila, F. Anselmet, T. Zhou, R. A. Antonia, J. Fluid Mech. 391 (1999) 359.

[4] R. A. Antonia, A. Chambers, L. Browne, Expts. in Fluids 1 (1983) 213.

[5] L. Mydlarski, Z. Warhaft, J. Fluid Mech. 320 (1996) 331-368.

[6] T. von Karman, L. Howarth, Proc. Roy. Soc. London A164 (1938) 192.

[7] P. G. Saffman (1968) 485-614.

[8] E. Lindborg, Phys. Fluids 11 (1999) 510.

[9] G. Batchelor, A. Townsend, Proc. Roy. Soc. London. Series A, Mathematical and Physical Sciences 190 (1947) 534-550.

[10] R. A. Antonia, M. Ould-Rouis, F. Anselmet, Y. Zhu, J. Fluid Mech. 332 (1997) 395.

[11] A. M. Yaglom, Dokl. Akad. Nauk SSSR 69 (1949) 743.

[12] L. Danaila, R. A. Antonia, P. Burattini, New J. Phys. 6(128) (2004) $1-23$.

[13] R. Hill, J. Fluid Mech. 353 (1997) 67.

[14] L. Danaila, F. Anselmet, T. Zhou, R. A. Antonia, J. Fluid Mech. 430 (2001) 87.

[15] G. Batchelor, Proc. Roy. Soc. 195 (1949) 513-532.

[16] B. K. Shivamoggi, R. A. Antonia, Fluid Dynamics Research 26 (2000) 95-104.

[17] S. Chandrasekhar, Philosophical Transactions of the Royal Society of London. Series A, Mathematical and Physical Sciences 242 (1950) 557577. 
[18] C. Cambon, L. Danaila, F. Godeferd, J. Scott, submitted to J. Fluid Mech. (2011) -.

[19] J.-F. Krawczynski, B. Renou, L. Danaila, Phys. Fluids 22 (2010) 1.

[20] J. Foucault, J. Carlier, M. Stanislas, Meas. Sci. Technol. 15 (2004) 10461058.

[21] V. Denshchikov, V. Kontratev, A. Romashev, Fluid Dyna. 6 (1978) 924926.

[22] J. Rolon, D. Veynante, J. Martin, Exp. Fluids 11 (1991) 313-324.

[23] L. Kostiuk, K. Bray, R. Cheng, Combust. Flame 92 (1993) 377-395.

[24] G. Stan, Fundamental characteristics of turbulent opposed impinging jets, Ph.D. thesis, U. Waterloo, Canada, 2000.

[25] C. Soong, P. Tzeng, C. Hsieh, Phys. Fluids 10 (1998) 2910-2921.

[26] A. Nahum, A. Seifert, Phys. Fluids 18 (2006) 064107.

[27] M. Barchillon, R. Curtet, J. Basic Eng. 86 (1964) 777-787.

[28] E. Lindborg, J. Fluid Mech. 302 (1995) 179-201.

[29] F. Anselmet, R. A. Antonia, M. Ould-Rouis, J. Turbulence 003 (2000) 1.

[30] M. Ould-Rouiss, Eur. Phys. J. B 15 (2000) 339-347.

[31] S. Galtier, Phys. Rev. E 80 (2009) 046301.

[32] F. S. Godeferd, C. Staquet, Journal of Fluid Mechanics 486 (2003) 115159.

[33] P. Sagaut, C. Cambon, Homogeneous turbulence dynamics, Cambridge University Press, 2008. 